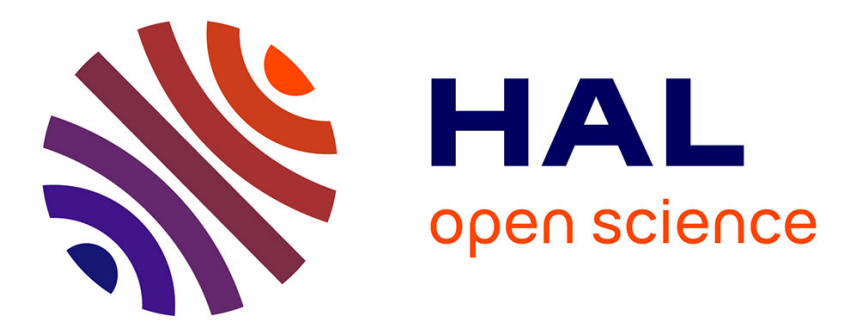

\title{
Effect of Manganese Doping of BaSrTiO 3 on Diffusion and Domain Wall Pinning
}

Kevin Nadaud, Caroline Borderon, Raphaël Renoud, Hartmut W. Gundel

\section{To cite this version:}

Kevin Nadaud, Caroline Borderon, Raphaël Renoud, Hartmut W. Gundel. Effect of Manganese Doping of BaSrTiO 3 on Diffusion and Domain Wall Pinning. Journal of Applied Physics, 2015, 117 (8), pp.084104. 10.1063/1.4913694 . hal-01121087

\section{HAL Id: hal-01121087 \\ https://hal.science/hal-01121087}

Submitted on 27 Feb 2015

HAL is a multi-disciplinary open access archive for the deposit and dissemination of scientific research documents, whether they are published or not. The documents may come from teaching and research institutions in France or abroad, or from public or private research centers.
L'archive ouverte pluridisciplinaire HAL, est destinée au dépôt et à la diffusion de documents scientifiques de niveau recherche, publiés ou non, émanant des établissements d'enseignement et de recherche français ou étrangers, des laboratoires publics ou privés. 


\title{
Effect of Manganese Doping of $\mathrm{BaSrTiO}_{3}$ on Diffusion and Domain Wall Pinning
}

\author{
Kevin Nadaud, ${ }^{\text {a) }}$ Caroline Borderon, ${ }^{\text {b) }}$ Raphaël Renoud, and Hartmut W. Gundel \\ IETR, UMR CNRS 6164, University of Nantes, Nantes, France
}

(Dated: February 27, 2015)

\begin{abstract}
In the present paper, the influence of manganese doping on the dielectric properties of $\mathrm{BaSrTiO}_{3}$ thin films is presented. The real and imaginary parts of the material's permittivity have been measured in a large frequency range $(100 \mathrm{~Hz}-$ $1 \mathrm{MHz}$ ) and as a function of the electric field. The tunability and the figure of merit of the material have been obtained from the measurement of the permittivity under an applied DC bias electric field. For the undoped material, the dielectric losses become important for a large DC bias which leads to breakdown. At a suitable dopant rate, this effect disappears. In order to better understand the origin of the related phenomena, we measure the permittivity as a function of the AC excitation amplitude and we decompose the obtained permittivity with the hyperbolic law. This enables to extract the different contributions of the bulk (low frequency diffusion and high frequency lattice relaxation) and of the domain wall motions (vibration and pinning/unpinning) to the material's dielectric permittivity and to understand the effect of manganese doping on each contribution. Knowledge of the related mechanisms allows us to establish the optimum dopant rate (mainly conditioned by the lattice contribution) and to reduce the domain wall motion, which finally is beneficial for the desired properties of the ferroelectric thin film. A particular attention is paid to low frequency diffusion, an especially harmful effect when a DC biasing is mandatory (tunable electronic component in mobile telecommunication devices for example).
\end{abstract}

Keywords: Ferroelectric, domain wall, doping, diffusion

\section{INTRODUCTION}

$\mathrm{Ba}_{(1-x)} \mathrm{Sr}_{x} \mathrm{TiO}_{3}(\mathrm{BST})$ is a mixed compound of $\mathrm{BaTiO}_{3}$ and $\mathrm{SrTiO}_{3}$ which is widely studied because its Curie temperature depends on the proportion between barium and strontium in the material. This allows us positioning the material in the structure-temperature phase diagram and to choose either the ferroelectric or the paraelectric phase state at room temperature ${ }^{1,2}$. As the polarization of ferroelectrics is a nonlinear function of the applied electric field, electrical controlling of the dielectric permittivity and hence realization of tunable microwave devices is possible ${ }^{3,4}$. For such applications, a large variation of the permittivity as a function of the applied field and low dielectric losses $\left(\tan \delta<10^{-2}\right)$ are needed.

The dielectric properties are strongly related to the quality of the material and more especially to the presence of defects. In ferroelectric thin films, oxygen vacancies are the predominant defects which participate to the conduction losses by releasing free electrons and generating dangling bonds in the material structure ${ }^{5,6}$. However, in order to minimize these losses, it is possible to perform doping of the material by substitution of one of the lattice ions by an ion of different valence. In the case of oxygen vacancies, electron acceptors, such as $\mathrm{Cr}^{2+7,8}, \mathrm{Mn}^{2+9,10}, \mathrm{Fe}^{2+11}$ or $\mathrm{Bi}^{3+12}$ are used.

In the present study, $\mathrm{Ba}_{0.80} \mathrm{Sr}_{0.20} \mathrm{TiO}_{3}$ thin films doped with manganese $\left(\mathrm{Mn}^{2+}\right)$ were realized by Chemical Solution Deposition (CSD) and spin-coating on alumina substrates using a multi-layer technique ${ }^{13}$. The manganese ions partially substitute the titanium ions and hence compensate the electrons released by the oxygen vacancies. The formation of oxygen

\footnotetext{
a)Electronic mail: kevin.nadaud@etu.univ-nantes.fr

b)Electronic mail: caroline.borderon@univ-nantes.fr
}

vacancies is probably facilitated by the conversion of $\mathrm{Ti}^{4+}$ to $\mathrm{Ti}^{3+}$, according to Du et al. ${ }^{14}$ and can be described using the Kröger-Vink formalism ${ }^{15}$ :

$$
2 \mathrm{Ti}_{\mathrm{Ti}} \longrightarrow 2 \mathrm{Ti}_{\mathrm{Ti}}^{\prime}+V_{\mathrm{O}}^{\bullet \bullet}
$$

Manganese ions are thus introduced in order to form a complex with the vacancies:

$$
\mathrm{Mn}_{\mathrm{Ti}}^{\prime \prime}+V_{\mathrm{O}}^{\bullet \bullet} \longrightarrow\left(\mathrm{Mn}_{\mathrm{Ti}}^{\prime \prime}-V_{\mathrm{O}}^{\bullet \bullet}\right)^{\times}
$$

The optimum dopant rate depends on the initial defect density in the material. In the present study, BST doping with 0 to $1.5 \%$ manganese has been performed and the thin film permittivity and the dielectric losses have been measured as a function of the frequency and of the applied electric field. The different contributions to the material's dielectric permittivity due to domain wall motion have been obtained from the hyperbolic law for the different dopant rates and as a function of the frequency. A compromise between a high permittivity and tunability on one hand and low losses on the other hand can be found.

\section{EXPERIMENTS}

The $\mathrm{Ba}_{0.80} \mathrm{Sr}_{0.20} \mathrm{TiO}_{3}$ (BST 80/20) thin films were realized by a sol-gel process based on the use of an alkoxide precursor. Barium acetate $\mathrm{Ba}\left(\mathrm{OOCCH}_{3}\right)_{2}$ was mixed with strontium acetate $\mathrm{Sr}\left(\mathrm{OOCCH}_{3}\right)_{2}$ in suitable proportions thus to obtain a $\mathrm{Ba} / \mathrm{Sr}$ ratio of $80 / 20$ and manganese acetate was added in order to realize up to $1.5 \%$ manganese doping of the BST. The powder was dissolved in acetic acid. The solution was heated at $100^{\circ} \mathrm{C}$ until complete dissolution and then cooled down to room temperature before the addition of the titanium 


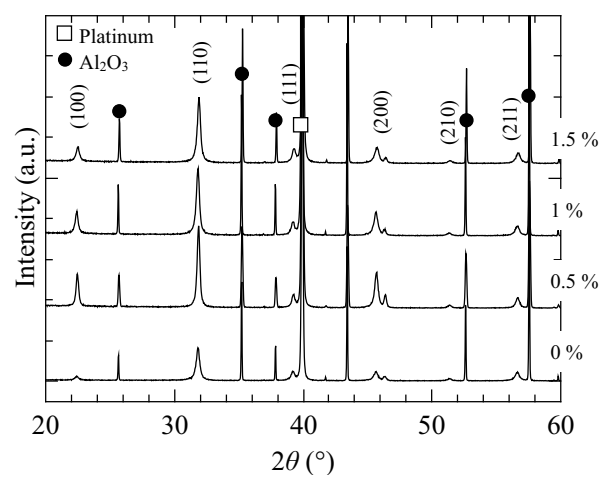

Figure 1. X-ray diffraction patterns of the BST(80/20) thin films on platinum coated alumina substrates for the different manganese dopant rates.

n-propoxide $\operatorname{Ti}\left(\mathrm{C}_{3} \mathrm{H}_{7} \mathrm{O}\right)_{4}$. The composition was adapted by taking into account the incorporation of $\mathrm{Mn}$ on the Ti sites in order to obtain a stoichiometric perovskite composition.

Ethylene glycol $\mathrm{HO}-\mathrm{CH}_{2}-\mathrm{CH}_{2}-\mathrm{OH}$ was added to maintain the atom mobility and to significantly reduce the appearance of cracks in the film ${ }^{16}$. Moreover, addition of ethylene glycol stabilizes the solution and prevents precipitation ${ }^{17}$.

Each solution was deposited on platinum coated alumina substrates at $4000 \mathrm{rpm}$ during $20 \mathrm{~s}$ and the samples were annealed during $15 \mathrm{~min}$ in a pre-heated open air furnace at $750^{\circ} \mathrm{C}$. Direct annealing (without a prior drying stage) was preferred in order to obtain a larger average grain size $e^{18}$ which is supposed to result in an increased permittivity of the material ${ }^{19,20}$. In this work, deposition of fourteen layers results in an overall film thickness of typically $0.85 \mu \mathrm{m}$.

The cross-sectional morphology of the films was examined with a Jeol 7600 scanning electron microscope (SEM). Xray diffraction (XRD) was performed for phase identification using a Siemens D5000 diffractometer with $\mathrm{CuK}_{\alpha}$ radiation. Platinum was deposited by RF sputtering in order to realize a MIM (Metal Insulator Metal) capacitor. The polarization versus electric field $P-E$ hysteresis loops were measured at $50 \mathrm{~Hz}$ using a Sawyer Tower circuit. The capacitance and the dielectric loss factor $(\tan \delta)$ were measured from $100 \mathrm{~Hz}$ to $1 \mathrm{MHz}$ with an AC field from $6 \mathrm{mV} / \mu \mathrm{m}$ to $1 \mathrm{~V} / \mu \mathrm{m}$ using an Agilent 4294A impedance meter and the dielectric permittivity of each sample was calculated from the measured capacitance.

\section{RESULTS AND DISCUSSION}

\section{A. Structural characterization}

The X-ray diffraction patterns of the BST thin films are shown in Fig. 1 for the different dopant rates. The (100), (110), (111), (200) and (211) peaks, corresponding to the perovskite structure of the material, are obtained, indicating that all BST films have a non-textured polycrystalline structure without preferential orientation. No parasitic peaks and no evidence for any secondary phase formation are visible.

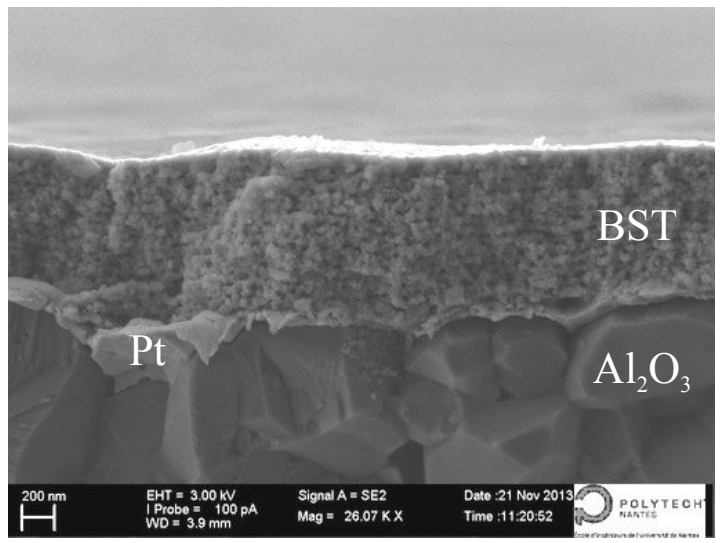

Figure 2. Typical cross section micrograph of the BST(80/20) thin films.

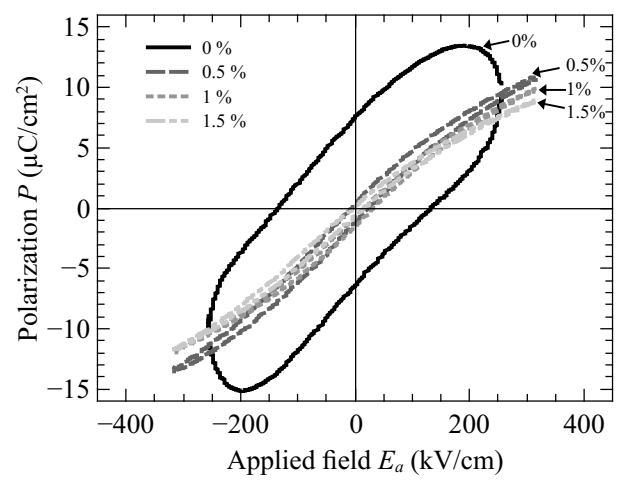

Figure 3. Hysteresis loops of the BST(80/20) films with different manganese dopant rates.

A typical cross section micrograph of the $\mathrm{Ba}_{0.80} \mathrm{Sr}_{0.20} \mathrm{TiO}_{3}$ film is shown in Fig. 2. All films are well-crystallized, have a uniform, dense and void-free microstructure without cracks and are composed of spherical grains. The average grain size is about $60 \mathrm{~nm}$ which corresponds to a single layer thickness of the BST film as crystallization is done layer by layer due to the direct annealing process.

\section{B. Dielectric characterization}

In order to verify the influence of the Mn doping effect on the ferroelectric properties, the $50 \mathrm{~Hz} P-E$ hysteresis loop of the BST thin films was measured at room temperature (Fig. 3). The undoped film has an open loop indicating important losses. The doped films show a rather slim ferroelectric loop with a very small coercive field and remnant polarization typical of the BST 80/20 composition. The effect of the manganese doping is clearly visible as a small amount of manganese is sufficient to considerably reduce the losses. For all the doped films, the polarization at saturation and the coercive field do not show a significant evolution: the values are approximately $11 \mu \mathrm{C} / \mathrm{cm}^{2}$ and $10 \mathrm{kV} / \mathrm{cm}$, respectively, which is similar to what is reported elsewhere ${ }^{21,22}$. This corresponds 


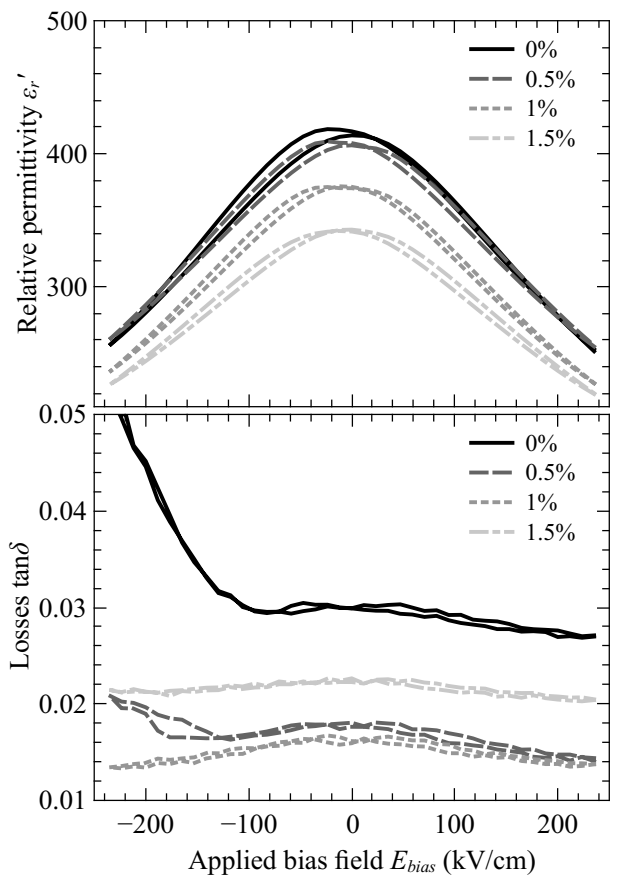

Figure 4. Relative permittivity and losses as a function of the applied bias field at $100 \mathrm{kHz}$ for the different manganese dopant rates.

to a soft material, easy to polarize/depolarize, with a large distribution of the coercive field which is centered on a relatively low value. This might be due to the crystallization of the BST in small spherical grains with different orientations.

The dielectric properties of the BST thin films at $100 \mathrm{kHz}$ as a function of the DC bias electric field are shown in Fig. 4. Addition of manganese affects the relative permittivity which decreases with increasing doping content. This is generally attributed to the increasing number of non-ferroelectric cells when incorporating the dopant into the material. Of course, this is probably not the only reason and further investigations are reported in section III C. The dielectric losses are significantly affected by the doping. Introduction of a small rate of manganese strongly reduces the dielectric losses until an optimum at $1 \%$ of Mn. In the case of the undoped material and for $0.5 \%$ manganese doping, the dielectric losses increase for the negative applied bias field which shows that charges accumulate at the electrodes, indicating that the doping is not sufficient in order to compensate the oxygen vacancies. Oxygen vacancies are polar defects which break the symmetry of the loop ${ }^{23,24}$ and doping hence improves the loop symmetry by compensating these vacancies. In the case of BST realized by the sol-gel method, the annealing probably leads to a significant concentration of oxygen vacancies at the interface between the ferroelectric and the bottom electrode. Contrary to the application of a negative field which allows a movement of these oxygen vacancies toward the top electrode, no migration is possible when the field is positive and no losses due to the diffusion are observed. Moreover, the diffusion of the oxygen vacancies, characterized by a complex conductivity, increases the values of the permittivity and of the losses and

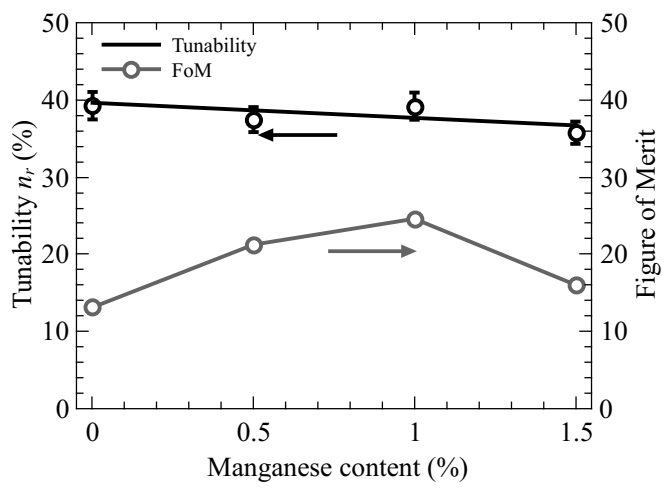

Figure 5. Tunability and figure of merit under $235 \mathrm{kV} / \mathrm{cm}$ at $100 \mathrm{kHz}$ and as a function of the manganese content.

a dissymmetry is thus observed for both loops. The diffusion losses are then more important as the applied field is high and this can lead to breakdown.

The tunability $n_{r}$ and the figure of merit at $100 \mathrm{kHz}$ of the BST thin films are given in Fig. 5. The tunability represents the variation of the permittivity under an applied DC electric field and has been evaluated with the usual definition ${ }^{25}$ :

$$
n_{r}=\frac{\varepsilon_{r}(0)-\varepsilon_{r}(E)}{\varepsilon_{r}(0)} \times 100,
$$

where $\varepsilon_{r}(0)$ and $\varepsilon_{r}(E)$ are the permittivity without and under the bias electric field $E$. For all dopant rates the tunability is around $38 \%$ under $235 \mathrm{kV} / \mathrm{cm}$, which is similar or higher than reported elsewhere ${ }^{13,26}$. The tunability slightly decreases with the dopant rate which might be due to the presence of an increasing number of non-ferroelectric cells containing manganese. The figure of merit $(F o M)$ is defined by:

$$
F O M=\frac{n_{r}}{\tan \delta_{E_{c}}(\%)},
$$

where $\tan \delta_{E_{c}}(\%)$ corresponds to the losses at the coercive field, given in percentage. The FoM reflects the fact that a tunable microwave component cannot take full advantage of a high tunability if the loss factor is important ${ }^{13}$. In our case, the FoM is highest for a dopant rate of $1 \%$ since the tunability almost does not vary and the losses are minimal. A manganese dopant rate of $1 \%$ hence offers the best compromise between tunability and losses. At $1.5 \%$ doping, the FoM decreases showing that the losses are more important due to supplementary defects created by the introduction of manganese.

The relative permittivity and the dielectric losses of the BST films as a function of frequency are presented in Fig. 6. As it has been shown before (cf. Fig 4), doping results in a decrease of the permittivity. However, it should be noted that the decay of permittivity with frequency is less pronounced in the case of $1 \%$ manganese doping. A similar evolution as a function of frequency can be observed for the dielectric losses. For $1 \% \mathrm{Mn}, \tan \delta$ is quasi-constant while it increases at low frequencies for the others dopant rates. This shows that there are diffusion phenomena that affect both, the value of the permittivity and the dielectric losses, also confirming what has been 


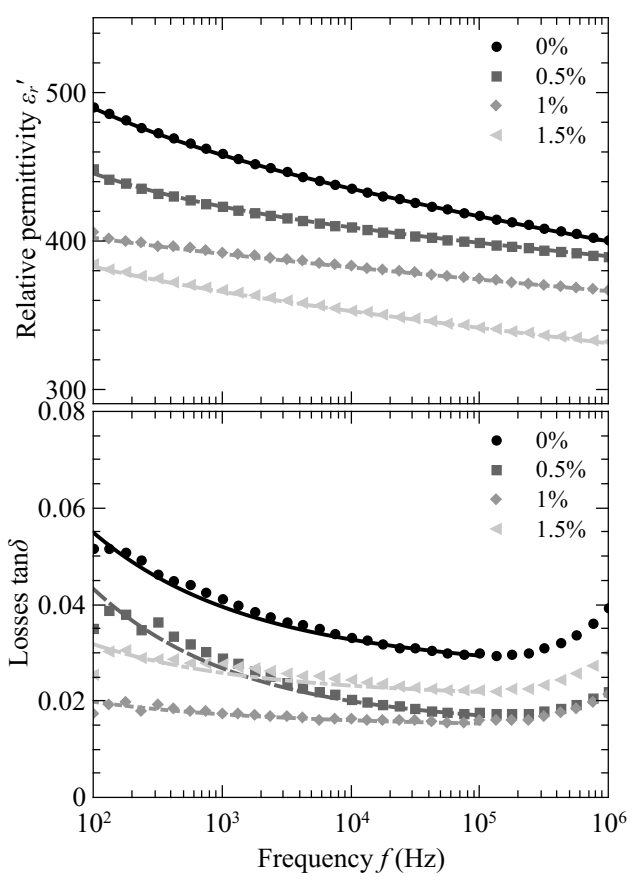

Figure 6. Relative permittivity and dielectric losses as a function of frequency for the different manganese dopant rates. The increase of the losses above $10^{5} \mathrm{~Hz}$ is artificial and due to a resonance of the impedance analyzer.

stated before (cf. Fig.4). A dopant rate of $1 \%$ of manganese is adequate to compensate the oxygen vacancies and hence establishes the best condition for applying the bias field. When the amount of the dopant becomes too important, the losses again increase because others defects are created.

In conclusion, the measurements show that the doping is necessary for reducing the low and high frequencies losses of the BST, however, it slightly reduces the material's tunability. For the optimum dopant rate, the frequency dependence of the dielectric properties is less pronounced. In order to have further analysis, it is necessary to identify the different parts of the permittivity. The hyperbolic law was hence used to dissociate the contributions of the lattice and of the vibrations and jumps of the domain walls. The contribution of the diffusion that appears at low frequencies was also taken into account.

\section{Study of the different permittivity contributions}

To determine the different contributions to the permittivity and the dielectric losses in the material, the complex permittivity as a function of the AC electric field has been analyzed. The dependence of the material's relative permittivity on the applied alternative field is shown in Fig. 7. In the case of ferroelectrics like BST(80/20), the hyperbolic law allows not only obtaining the Rayleigh coefficient but also the threshold field and the permittivity due to the domain wall vibrations ${ }^{27-29}$. This is not the case of a polynomial development which is sometimes used and where the coefficients

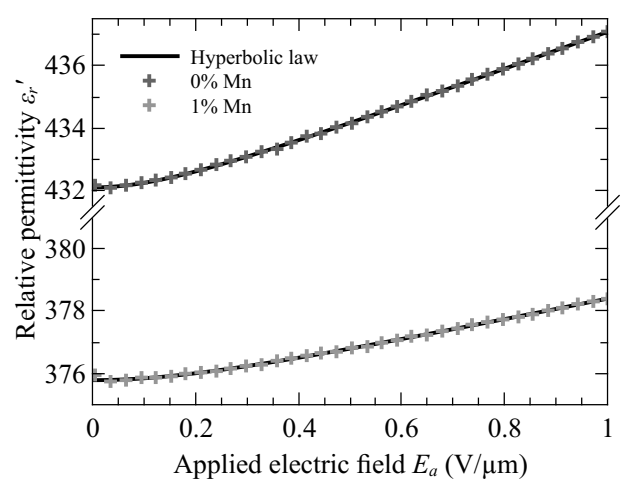

Figure 7. Examples of the hyperbolic law fits at $10 \mathrm{kHz}$ for undoped BST and for a $1 \%$ manganese dopant rate.

of the polynomial do not have a simple relationship with the Rayleigh coefficient ${ }^{27,29}$. If we consider the amplitude of this field $E_{a}$, the permittivity can be described by: ${ }^{27}$

$$
\varepsilon_{r}=\varepsilon_{r l}+\sqrt{\varepsilon_{r-r e v}^{2}+\left(\alpha_{r} E_{a}\right)^{2}},
$$

where $\varepsilon_{r l}$ corresponds to the lattice contribution, $\varepsilon_{r \text {-rev }}$ to the contribution due to domain wall vibrations (also called reversible contribution) and $\alpha_{r}$ to the contribution due to domain wall pinning/unpinning (also called irreversible contribution). $\varepsilon_{r \text {-rev }}$ and $\alpha_{r}$ depend on the crystal structure but $\alpha_{r}$ also reflects the presence of impurities, dopants or defects ${ }^{30}$. The different parameters also depend on the frequency ${ }^{28}$. The decomposition of the complex permittivity is shown in Fig. 8 for the different dopant rates and as a function of the frequency.

The most important contribution to the permittivity comes from the lattice $\varepsilon_{r l}$ (Fig. 8a) and shows the same evolution as the overall permittivity (Fig. 6). According to Joncher ${ }^{31}$, far away from a relaxation, the permittivity varies following a power law:

$$
\varepsilon_{r l}=\varepsilon_{h f} \omega^{s_{h f}}+\varepsilon_{l f} \omega^{s_{l f}},
$$

where $\varepsilon_{h f} \omega^{s_{h f}}$ and $\varepsilon_{l f} \omega^{s_{l f}}$ represent respectively the high frequency relaxation and the low frequency diffusion ${ }^{31}$. Fitting of the experimental data (standard error of least squares method) is presented in Fig. 8a and the coefficients are given in Table I.

According to the Kramer-Kronig relations (invariance of a power law by K.-K. relations), the exponents of the two contributions are the same for the real and the imaginary part of the permittivity, only the weighting coefficients vary. The exponent $s_{h f}$, representing the high frequency decrease of the permittivity, goes through a minimum for a dopant rate of $1 \%$. This signifies that the properties of the film with $1 \%$ manganese are more stable in frequency than for the other dopant rates. On the contrary, $s_{l f}$, the exponent representing the low frequency diffusion, is identical for all dopant rates which indicates that the same conduction phenomenon is present. In this case, it may be diffusion of oxygen vacancies. For diffusion, the value of $s_{l f}$ theoretically established by Jonscher is -0.5 and does not depend on the carrier concentration ${ }^{32}$. In 


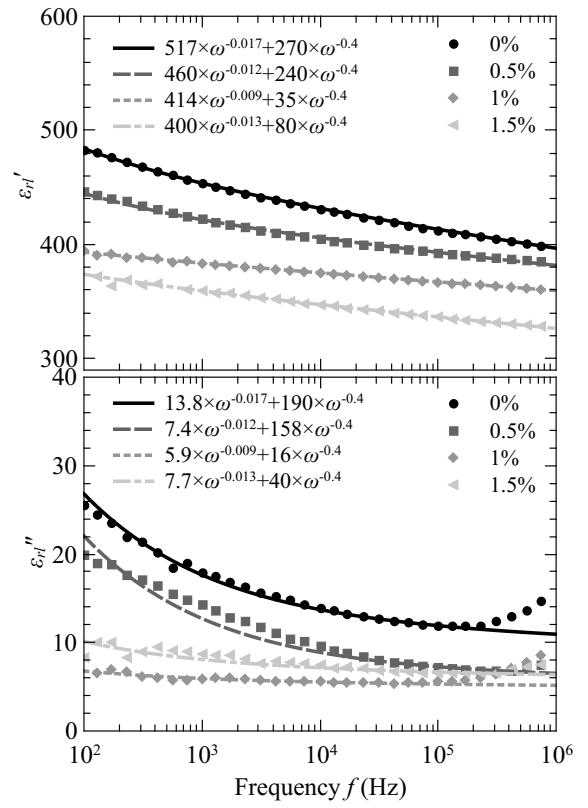

(a)

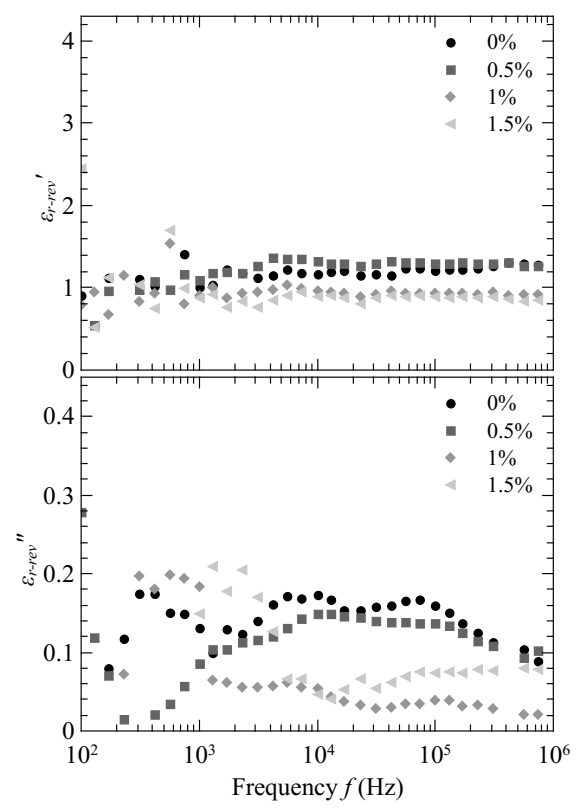

(b)

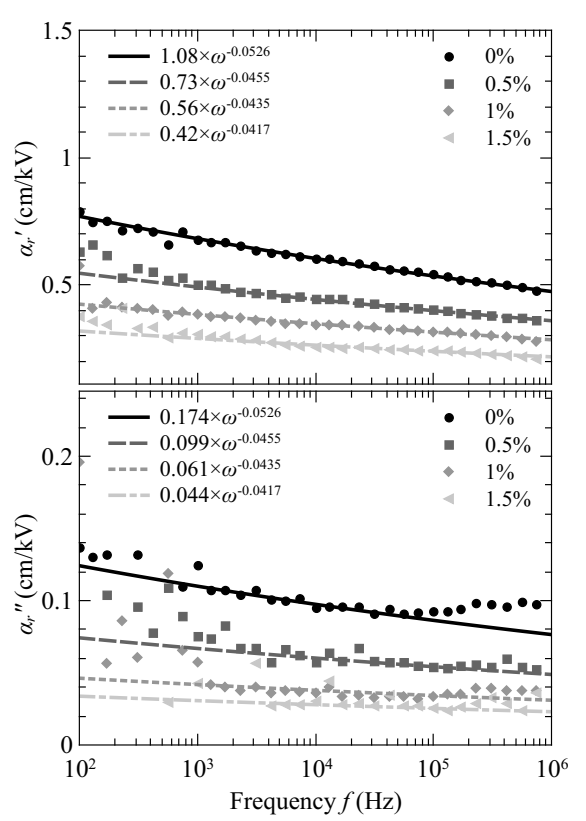

(c)

Figure 8. Real and imaginary part of the permittivity as a function of frequency, decomposed according to the hyperbolic law, showing the bulk contribution (a), and the contributions of domain wall vibrations (b) and domain wall displacements (c). The increase of the imaginary part above $10^{5} \mathrm{~Hz}$ is artificial and due to a resonance of the impedance analyzer.

Table I. Exponents, weighting coefficients and dissipation factors of the different lattice contributions to the permittivity.

\begin{tabular}{|c|c|c|c|c|c|c|c|c|}
\hline \multirow{2}{*}{ dopant rate } & \multicolumn{4}{|c|}{ High frequency relaxation } & \multicolumn{4}{|c|}{ Low frequency diffusion } \\
\hline & $s_{h f}$ & $\varepsilon_{h f}^{\prime}$ & $\varepsilon_{h f}^{\prime \prime}$ & $\tan \delta_{h f}$ & $s_{l f}$ & $\varepsilon_{l f}^{\prime}$ & $\varepsilon_{l f}^{\prime \prime}$ & $\tan \delta_{l f}$ \\
\hline $0 \%$ & -0.017 & 517 & 13.8 & 0.0263 & -0.4 & 270 & 190 & 0.704 \\
\hline $0.5 \%$ & -0.012 & 461 & 7.4 & 0.0160 & -0.4 & 240 & 158 & 0.658 \\
\hline $1 \%$ & -0.009 & 414 & 5.9 & 0.0143 & -0.4 & 35 & 16 & 0.457 \\
\hline $1.5 \%$ & -0.013 & 400 & 7.7 & 0.0196 & -0.4 & 80 & 40 & 0.500 \\
\hline
\end{tabular}

our case, the obtained value is -0.4 . The difference between the theoretical and experimental values could be due to the polycrystalline character of the BST studied (change in the direction of the crystallographic axes from a grain to another), that differs from the ideal case used by Jonscher. Contrary to $s_{l f}$, the prefactor $\varepsilon_{l f}$ is a function of the carrier concentration.

In order to study the evolution of the dissipation factor of the high and low frequency components of the lattice contribution, $\tan \delta_{h f}=\varepsilon_{h f}^{\prime \prime} / \varepsilon_{h f}^{\prime}$ and $\tan \delta_{l f}=\varepsilon_{l f}^{\prime \prime} / \varepsilon_{l f}^{\prime}$ have been calculated and are reported in Table I. When the dopant rate increases, the high frequency losses $\tan \delta_{h f}$ decrease because the introduction of the manganese compensates oxygen vacancies in the lattice. If doping becomes too important (1.5\%), the losses increase due to the creation of additional defects. The coefficients representing the low frequency diffusion $\varepsilon_{l f}^{\prime}$ and $\varepsilon_{l f}^{\prime \prime}$ are quite important for the undoped and the $0.5 \%$ doped films, especially for the losses. In the case of $1 \% \mathrm{Mn}$ doping the coefficient representing the diffusion becomes very small and hence only little diffusion is observed. It is im- portant to note that $\varepsilon_{l f}^{\prime \prime}$ is more than ten times smaller for this dopant rate than for the undoped and $0.5 \%$ doped film. This explains the observed high losses under bias field when the amount of doping is too low (Fig. 4). For the dopant rate of $1.5 \%$, the diffusion losses again slightly increase due to the addition of defects, similar to what is observed for the high frequency contribution. The dissipation factor value is very important which shows that the diffusion is a very dissipative phenomenon. Again the minimum of the losses appears for a dopant rate of $1 \%$.

In conclusion on the influence of Mn doping on the lattice contribution to the material's permittivity, one can note that the dissipation factor value $\tan \delta_{l f}$ is very important which shows that diffusion is a very dissipative phenomenon. Addition of manganese compensates the oxygen vacancies present in the material and hence reduces the low frequency diffusion. The dopant rate has to be sufficient in order to compensate all the vacancies but should not be too important such as to create new additional defects. The minima of the coefficients are observed at a dopant rate of $1 \% \mathrm{Mn}$ which seems to be optimum 
Table II. Exponents, weighting coefficients and dissipation factors of the domain wall pinning/unpinning contribution.

\begin{tabular}{ccccc}
\hline \hline dopant rate & $s_{\alpha}$ & $\alpha_{f}^{\prime}$ & $\alpha_{f}^{\prime \prime}$ & $m_{\alpha}$ \\
\hline $0 \%$ & -0.0526 & 1.08 & 0.174 & 0.161 \\
$0.5 \%$ & -0.0455 & 0.73 & 0.099 & 0.136 \\
$1 \%$ & -0.0435 & 0.56 & 0.061 & 0.109 \\
$1.5 \%$ & -0.0417 & 0.42 & 0.044 & 0.104 \\
\hline \hline
\end{tabular}

for reducing high frequency losses and low frequency diffusion. Moreover, the frequency dependence of the material's properties is lowest at this dopant rate.

Compared to the lattice part, the contribution of the domain walls, vibrations (Fig. 8b) and irreversible displacements (Fig. 8c), to the material's permittivity are small. As a consequence, the contribution due to domain wall vibrations $\varepsilon_{r \text {-rev }}$ is quite difficult to obtain and its evolution with the dopant rate is not clearly visible in Fig. 8b. The portion of the domain wall vibrations on the overall permittivity is below $0.5 \%$. The parameters $\alpha_{r}$, describing the irreversible displacement (Fig. 8c) are approximately 0.2 to $0.8 \mathrm{~cm} / \mathrm{kV}$ for the real part and 0.02 to $0.15 \mathrm{~cm} / \mathrm{kV}$ for the imaginary part which is approximately ten times smaller than reported for other materials like $\mathrm{PZT}^{33}$ or $\mathrm{BZT}^{34}$. In comparison, the contribution of irreversible domain wall displacements to the total dielectric permittivity $\left(\alpha_{r} E_{a} / \varepsilon_{r}\right)$ is approximately $21 \%$ at $30 \mathrm{kV} / \mathrm{cm}$ for a PZT thin film ${ }^{33}$ whereas it is only $5 \%$ in the case of the BST $(80 / 20)$. The total contribution of domain walls is thus very low for BST(80/20) compared to what is found for PZT. As a consequence, the influence on the domain wall behavior from doping is not observable on the hysteresis loops shown in Fig. 3 and only the contribution of diffusion and of the lattice are visible. The concentration of domain walls seems to be low which might be due to the small grain size in the BST thin film.

The manganese doping also affects the domain wall displacement facility since the irreversible part decreases with increasing doping (Fig. 8c). This irreversible part seems to decrease with a power law of the frequency following the equation:

$$
\alpha_{r}=\alpha_{f} \omega^{s_{\alpha}},
$$

where $\alpha_{f}$ represents the irreversible contribution at $\omega=$ $1 \mathrm{rad} / \mathrm{s}$ and $s_{\alpha}$ indicates the frequency dependence of $\alpha_{r}$. Likewise the case of the lattice contribution, the same exponent has been used for fitting the real and the imaginary parts in order to satisfy the K.-K. relations. The logarithm law as proposed by Taylor ${ }^{33}$ has not been chosen because according to the K.$\mathrm{K}$. relations, the imaginary part has to be constant if the real part follows a logarithm decrease, which is not the case here. Nevertheless, as the frequency dependence is very small, both laws are very close to each other.

The irreversible domain wall contribution coefficient $\alpha_{r}$ has been fitted and the real and imaginary coefficients $\alpha_{f}^{\prime}$ and $\alpha_{f}^{\prime \prime}$ show the same evolution as a function of the dopant rate (inset of Fig. 9). The inverse of $\alpha_{f}^{\prime}$ and $\alpha_{f}^{\prime \prime}$ increases linearly

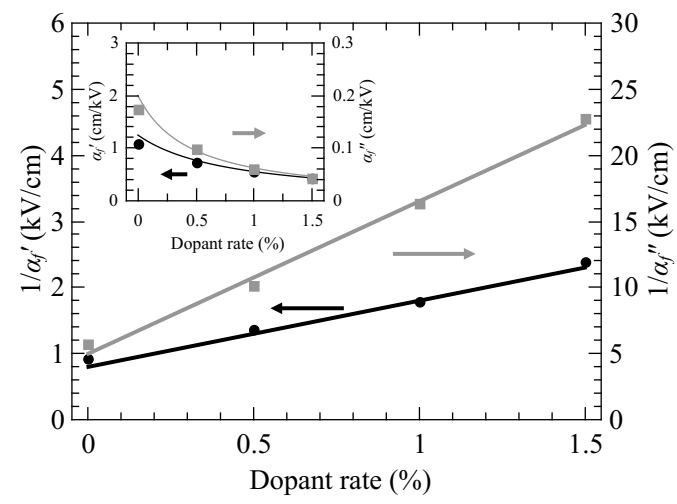

Figure 9. Inverse of the coefficients $\alpha_{f}^{\prime}$ and $\alpha_{f}^{\prime \prime}$ obtained by fitting of the irreversible part of the permittivity.

with the Mn doping (Fig. 9). This is in agreement with the statistical theory of Boser ${ }^{30}$ and shows that manganese acts as a pinning center and represents an obstacle to large distance domain wall motion ${ }^{35}$. As a consequence, the domain wall mobility and hence the contribution of the domain walls is reduced and the permittivity and the tunability of the material become smaller.

The dissipation factor $m_{\alpha}=\alpha_{f}^{\prime \prime} / \alpha_{f}^{\prime}$ of the domain wall pinning/unpinning has been computed and is reported in Table II. The obtained values are lower than predicted by the Rayleigh model $\left(m_{\alpha} \approx 0.5\right)$ and what has already been reported elsewhere ${ }^{36}$. As the wall density is small, there is only little interaction between the walls and the losses associated to $\alpha_{r}$ are low. The dissipation factor also decreases with the amount of doping showing that the phenomenon becomes less dissipative. This is due to the fact that the pinning centers are deeper and the domain structure is stabilized ${ }^{37}$, which results in less losses. The frequency dependence of the irreversible domain wall contribution, represented by $s_{\alpha}$, also decreases with the addition of manganese as reported before ${ }^{35}$. This may be due to the fact that the introduction of a large number of acceptors defects stabilize the domain walls, the relaxation associated to domain wall pinning appears then at lower frequencies ${ }^{38}$.

Finally, all the results from the domain wall characterizations indicate that the manganese doping leads to a hardening of the BST(80/20): the coefficient $\alpha_{f}^{\prime}$ associated to domain wall pinning decreases when increasing the dopant rate and the pinning losses are reduced which correspond to a stabilization of the walls. Similar results are reported for PZT when an acceptor dopant is used in order to increase its hardness ${ }^{37}$. The main difference is that BST(80/20) is initially a soft material with a low domain wall density.

\section{CONCLUSION}

Ion substitution at the $\mathrm{B}$-site of the perovskite lattice of $\mathrm{Ba}_{0.80} \mathrm{Sr}_{0.20} \mathrm{TiO}_{3}$ (BST 80/20) thin films by $\mathrm{Mn}^{2+}$ ions has been investigated in order to study the effect of the doping on the dielectric properties. The BST $80 / 20$ composition is 
located near to the ferroelectric-paraelectric structural phase boundary and hence has a high tunability. Introduction of a small amount of the manganese dopant prevents diffusion due to oxygen vacancies since manganese is an electron acceptor and hence considerable reduction of the dielectric losses is observed. As the manganese substitution creates non ferroelectric cells, the permittivity and tunability of the thin film, however, slightly decrease. At higher manganese content, additional defects are created in the material and the diffusion losses rise again. Doping with $1 \%$ manganese results in the best figure of merit.

Utilization of the hyperbolic law allows us to discern the different components of the material's complex permittivity. Thus it is shown that the contributions of domain wall motion (vibrations and displacements) are rather small compared to the lattice contribution. By dissociating the two contributions to the lattice permittivity, we show that an appropriate dopant rate considerably reduces low frequency diffusion (by a factor higher than ten) while diminishing high frequency losses at the same time. The addition of manganese also reduces the domain wall mobility by the creation of additional pinning centers and hence the contribution to the complex permittivity and tunability. The doping corresponds to a hardening of the BST since the domain structure is stabilized, the domain wall pinning/unpinning is limited and the associated losses are reduced. Therefore reducing the domain wall contribution is then beneficial for decreasing the material's dielectric losses since wall motion is a very dissipative phenomena. Doping with $1 \%$ of manganese thus is a compromise between reducing the domain wall contribution (constituting only a small part of the permittivity) and maintaining a high tunability and low dielectric losses due to the lattice contribution which is responsible for the predominant part of the material's overall properties.

Moreover, it has been shown that the dielectric properties of the BST thin films are rather stable over many frequency decades and no increase of the losses at low frequencies is observed at the optimum dopant rate of $1 \%$ manganese. Limiting the low frequency diffusion losses is essential in order to prevent breakdown when a DC field is applied. This is particularly important for the use of ferroelectric materials in tunable microwave components where a high tunability and low losses are required and stability of the dielectric properties is mandatory.

\section{ACKNOWLEDGEMENTS}

The authors would like to thank S. Ginestar for the realization of the SEM image. The financial support of the CNRS (Centre National de Recherche Scientifique) and the French Region "Pays de la Loire" is gratefully acknowledged.

\section{REFERENCES}

${ }^{1}$ S. Lahiry and A. Mansingh, Ferroelectrics 329, 39 (2005).
${ }^{2}$ B. Baumert, L.-H. Chang, A. Matsuda, T.-L. Tsai, C. J. Tracy, R. Gregory, P. Fejes, N. Cave, W. Chen, D. Taylor, T. Otsuki, E. Fujii, S. Hayashi, and K. Suu, Journal of Applied Physics 82, 2558 (1997).

${ }^{3}$ H. Gundel, R. Renoud, C. Borderon, S. Pavy, A. Sharaiha, V. H. Nguyen, $\mathrm{R}$. Benzerga, and C. Delaveaud, in IEEE International Symposium on the Applications of Ferroelectrics (2012) pp. 1-4.

${ }^{4}$ K. Nadaud, R. Gillard, E. Fourn, H. Gundel, and C. Borderon, in Loughborough Antennas and Propagation Conference (2014) pp. 214-217.

${ }^{5}$ G. Choi, H. Tuller, and D. Goldschmidt, Physical Review B 34, 6972 (1986).

${ }^{6}$ M.-C. Chiu, Y.-C. Lee, and F.-S. Shieu, Journal of The Electrochemical Society 152, 194 (2005).

${ }^{7}$ J. Kim, S. Kim, W. Kim, T. Ha, I.-S. Kim, J.-S. Song, R. Guo, and A. Bhalla, Materials Letters 60, 2322 (2006).

${ }^{8}$ K.-T. Kim. and C.-I. Kim., Thin Solid Films 472, 26 (2005).

${ }^{9}$ L. Mingli, X. Mingxia, L. Hui, L. Xiaolei, and X. Tingxian, Acta PhysicoChimica Sinica 24, 1405 (2008).

${ }^{10}$ M. Jain, S. Majumder, R. Katiyar, F. Miranda, and F. Van Keuls, Applied Physics Letters 82, 1911 (2003).

${ }^{11}$ W. Hofman, S. Hoffmann, and R. Waser, Thin Solid Films 305, 66 (1997).

${ }^{12}$ K.-T. Kim. and C.-I. Kim., Microelectronic Engineering 66, 835 (2003).

${ }^{13}$ C. Borderon, D. Averty, R. Seveno, and H. W. Gundel, Integrated Ferroelectrics 97, 12 (2008).

${ }^{14}$ X. Du and I.-W. Chen, Journal of Applied Physics 83, 7789 (1998).

${ }^{15} \mathrm{~B}$. Mitchell, An Introduction to Materials Engineering and Science for Chemical and Materials Engineers (Wiley, 2004).

${ }^{16} \mathrm{~N}$. Giridharan, S. Madeswaran, and R. Jayavel, Journal of Crystal Growth 237-239, Part 1, 468 (2002).

${ }^{17}$ H.-Y. Tian, W.-G. Luo, X.-H. Pu, X.-Y. He, P.-S. Qiu, and A.-L. Ding, Materials Chemistry and Physics 69, 166 (2001).

${ }^{18}$ R. Schwartz, T. Schneller, and R. Waser, Comptes Rendus Chimie 7, 433 (2004).

${ }^{19}$ S. Hoffmann and R. Waser, Journal of the European Ceramic Society 19, 1339 (1999).

${ }^{20}$ U. Hasenkox, S. Hoffmann, and R. Waser, Journal of Sol-Gel Science and Technology 12, 67 (1998).

${ }^{21}$ J.-G. Cheng, X.-J. Meng, B. Li, J. Tang, S.-L. Guo, J.-H. Chu, M. Wang, H. Wang, and Z. Wang, Applied Physics Letters 75, 2132 (1999).

${ }^{22}$ T.-J. Zhang, H. Ni, and W. Wang, Journal of Materials Synthesis and Processing 10, 17 (2002).

${ }^{23}$ G. Le Rhun, R. Bouregba, and G. Poullain, Journal of Applied Physics 96, 5712 (2004).

${ }^{24}$ W. Wu, K. H. Wong, and C. L. Choy, Applied Physics Letters 85, 5013 (2004).

${ }^{25}$ D. Dimos, M. Raymond, R. Schwartz, H. Al-Shareef, and C. Mueller, Journal of Electroceramics 1, 145 (1997).

${ }^{26}$ C. Borderon, D. Averty, R. Seveno, and H. W. Gundel, Ferroelectrics 362, 1 (2008).

${ }^{27}$ C. Borderon, R. Renoud, M. Ragheb, and H. W. Gundel, Applied Physics Letters 98, 112903 (2011).

${ }^{28}$ R. Renoud, C. Borderon, and H. Gundel, Ultrasonics, Ferroelectrics, and Frequency Control, IEEE Transactions on 58, 1975 (2011).

${ }^{29} \mathrm{C}$. Borderon, R. Renoud, and H. Gundel, in IEEE International Symposium on the Applications of Ferroelectrics (2010) pp. 1-4.

${ }^{30} \mathrm{O}$. Boser, Journal of Applied Physics 62, 1344 (1987).

${ }^{31}$ A. K. Jonscher, Journal of Physics D: Applied Physics 32, R57 (1999).

${ }^{32}$ A. Jonscher, Dielectric relaxation in solids (Chelsea Dielectrics Press Ltd, 1983).

${ }^{33}$ D. V. Taylor and D. Damjanovic, Journal of Applied Physics 82, 1973 (1997).

${ }^{34}$ J. Miao, J. Yuan, H. Wu, S. B. Yang, B. Xu, L. X. Cao, and B. R. Zhao, Applied Physics Letters 90, 022903 (2007).

${ }^{35}$ W. Zhu, I. Fujii, W. Ren, and S. Trolier-McKinstry, Journal of Applied Physics 109, 064105 (2011).

${ }^{36}$ J. E. García, R. Pérez, and A. Albareda, Journal of Physics: Condensed Matter 17, 7143 (2005).

${ }^{37}$ D. Damjanovic, Reports on Progress in Physics 61, 1267 (1998).

${ }^{38}$ C. Borderon, R. Renoud, M. Ragheb, and H. W. Gundel, Applied Physics Letters 104, 072902 (2014). 